\title{
The Role of Dilation and Internal Urethrotomy as a Risk Factor of Failure in Patients Who Undergoing One-Stage Bulbar Oral Graft Urethroplasty
}

\author{
Guido Barbagli ${ }^{1}$, Giorgio Guazzoni ${ }^{2}$, Salvatore Sansalone ${ }^{1}$, Giuseppe Romano ${ }^{1}$, Massimo Lazzeri ${ }^{*}$ \\ ${ }^{1}$ Center for Reconstructive Urethral Surgery, Arezzo, Italy \\ ${ }^{2}$ Department of Urology, University-Hospital San Raffaele, Milan, Italy \\ ${ }^{3}$ Clinical Research Institute "Prof. M. Fanfani", Florence, Italy \\ Email: *lazzeri.maximus@gmail.com
}

Received November 27, 2011; revised January 18, 2012; accepted January 28, 2012

\begin{abstract}
Purpose: To test the hypothesis if dilation or direct visual internal urethrotomy (DVIU) are predictive of urethroplasty failure. Retrospective study, from 1999 to 2010, including 184 patients (median age 37 years) who underwent ventral onlay oral graft urethroplasty for bulbar strictures. Exclusion criteria were traumatic strictures, lichen sclerosus, failed hypospadias repair, failed urethroplasty, panurethral strictures, and incomplete medical charts. Pre-operative evaluation included clinical history, physical examination, urine culture, residual urine measurement, uroflowmetry, urethrography, ultrasound and urethroscopy. Surgery was considered a failure when any post-operative instrumentation was needed. Median follow-up was 48 months. Out of 184 patients, 38 (20.7\%) had not undergone previous treatment, 7 (3.8\%) had undergone dilation, $81(44 \%)$ DVIU and 58 (31.5\%) DVIU associated with dilation. Out of 184 patients, 157 (85.3\%) were successful and $27(14.7 \%)$ failures. Out of 38 patients who had not undergone previous treatment, $33(86.8 \%)$ were successful; out of 7 patients who had undergone dilation, $6(85.7 \%)$ were successful; out of 81 patients who had undergone DVIU, 72 (88.9\%) were successful; out of 58 patients who had undergone DVIU and dilation, 46 (79.3\%) were successful. According to the number of previous DVIU, ventral graft urethroplasty for bulbar strictures showed high failure rate in patients who had undergone more than four DVIU associated or not with dilation.
\end{abstract}

Keywords: Urethral Stricture; Direct Vision Internal Urethrotomy; Dilation; Urethroplasty

\section{Introduction}

Reconstructive urethral surgery has greatly improved in both safety and effectiveness in the last 10 years and urethral stricture should be now considered an open surgical disease [1]. Dilation and direct vision internal urethrotomy (DVIU) are now regarded as neither cost-effective nor efficacious as a long-term strategy [1-3]. In 2010, Santucci and Eisemberg evaluated the success rate of DVIU in a series of 76 patients with simple urethral strictures and concluded that the success rates were no higher than $9 \%$ for the first or subsequent urethrotomy during the observation period [4]. However, dilation and DVIU are the most common procedures used by the majority of urologists in the United States [5-7]. Recently, several authors analyzed the trends in male urethral stricture management in the United States using the data from the 1992-2001 Medicare claims [8]. These authors concluded that despite the poor overall efficacy of dilation and DVIU, urethroplasty rates were the lowest of all

${ }^{*}$ Corresponding author. treatments [8]. In 1996, 1997, 1998, three articles in the literature, including one on a large series of patients, showed that repeated dilation or DVIU are not clinically effective [9-11]. For many years, these three articles were considered and quoted in the literature as fundamental milestones on this topic. In 2004, Greenwell et al. emphasized that repeated dilation and DVUI are neither clinically effective nor cost-effective [12]. The best way to primarily treat short bulbar urethral strictures, using DVIU or open urethroplasty, represents a controversial issue in the literature. Wright et al. suggested that the most cost-effective strategy for the management of short bulbar urethral strictures is to reserve urethroplasty for patients in whom a single DVIU has failed [13]. On the contrary, Rourke and Jordan suggested that treatment for short bulbar urethral strictures with primary reconstruction is less costly than treatment with DVIU [14]. Should repeated failed dilation or DVIU be considered a risk factor for the outcome of urethroplasty? Literature on this topic is found to be lacking. Successful posterior 
urethroplasty after pelvic trauma has been noted to decrease greatly if there was previous urethral manipulation [15]. On the contrary, oral graft urethroplasty for anterior urethral strictures has not been shown to significantly lead to poorer outcome if previous DVIU was unsuccessful [16]. Recently, Breyer et al. studied the patient risk factors that promote urethroplasty failure in a cohort of 443 patients [17]. These authors found that stricture length (greater than $4 \mathrm{~cm}$ ) and prior failed DVIU or urethroplasty are predictive of failure after urethroplasty [17]. We retrospectively reviewed a large and homogeneous series of patients who underwent ventral onlay graft urethroplasty for bulbar urethral strictures to investigate if previous failed dilation or DVIU were predictive of urethroplasty failure.

\section{Methods}

The current study is an observational, descriptive and retrospective study of consecutive adult male patients evaluated and treated for bulbar urethral strictures. Study inclusion criteria were: male patient aged 14 to 80 years who had undergone ventral onlay oral graft bulbar urethroplasty.

Exclusion criteria were traumatic strictures, pan-urethral strictures, malignant urethral lesions, previous failed urethroplasty, genital lichen sclerosus, failed hypospadias repair and incomplete data on personal medical charts. All data from May 1999 until June 2010 were retrospecttively collected.

Pre-operative evaluation included clinical history, physical examination, urine culture, residual urine measurement, uroflowmetry, retrograde and voiding cystourethrography, urethral ultrasound and urethroscopy. All patients underwent ventral onlay oral graft bulbar urethroplasty by a single surgeon (GB), without significant changes in our standard technique [18]. Patients were discharged from the hospital 3 days after surgery and voiding cystourethrography was performed 3 weeks later. Clinical outcome was considered a failure when any postoperative instrumentation was needed, including dilation. Uroflowmetry and urine culture were repeated every 4 months in the first year and annually thereafter. When symptoms of decreased force of stream were present and the uroflowmetry was less than $12 \mathrm{ml}$ per second, urethrography, urethral ultrasound and urethroscopy were repeated. Median follow-up was 48 months (range 12 to 145 months).

The study design allowed us to evaluate if repeated failed dilation and DVIU were predictive of failure after ventral onlay oral graft bulbar urethroplasty. As the study was an investigative retrospective observational analysis and the sample size of some sub-groups was very small, comparative predictive statistics were not performed. Descriptive statistical analysis was used.

\section{Results}

Between May 1999 and June 2010, 184 patients underwent one-stage ventral onlay oral graft urethroplasty for bulbar urethral strictures. Median patient age was 37 years (range 14 to 80 years).

Stricture length was $1-2 \mathrm{~cm}$ in 3 patients $(1.6 \%), 2$ - 3 $\mathrm{cm}$ in $9(4.9 \%), 3-4 \mathrm{~cm}$ in $33(17.9 \%), 4-5 \mathrm{~cm}$ in 90 $(48.9 \%), 5-6 \mathrm{~cm}$ in $43(23.4 \%)$, and $>6 \mathrm{~cm}$ in $6(3.3 \%)$ patients. Stricture etiology was unknown in $146(79.4 \%)$ patients, catheter in $22(11.9 \%)$, instrumentation in 13 (7.1), infection in 3(1.6\%).

Out of 184 patients, $38(20.7 \%)$ had not undergone previous treatment, $7(3.8 \%)$ had undergone periodic dilation, 81 (44\%) DVIU and 58 (31.5\%) DVIU associated with periodic dilation (Table 1).

Out of 184 patients, $157(85.3 \%)$ were successful and $27(14.7 \%)$ failures (Table 1). Out of 38 patients who had not undergone previous treatment before the urethroplasty, $33(86.8 \%)$ were successful and 5 (13.2\%) failures; out of 7 patients who had undergone dilation, 6 $(85.7 \%)$ were successful and 1 (14.3\%) failure; out of 81 patients who had undergone DVIU, 72 (88.9\%) were successful and $9(11.1 \%)$ failures; out of 58 patients who had undergone DVIU and dilation, 46 (79.3\%) were successful and $12(20.6 \%)$ failures (Table 1). The success rate according to the number of previous DVIU (from 1 to 15 ) is summarized in Table 2, and the success rate according to the number of previous DVIU (from 1 to 10) and dilation in Table 3.

\section{Discussion}

The role of failed previous urethral manipulation using

Table 1. Previous treatment and success rate.

\begin{tabular}{lccc}
\hline Previous treatment & N. patients & Success rate \% & Failure rate \% \\
\hline None & $38(20.7 \%)$ & $33(86.8 \%)$ & $5(13.2 \%)$ \\
Dilation & $7(3.8 \%)$ & $6(85.7 \%)$ & $1(14.3 \%)$ \\
DVIU & $81(44 \%)$ & $72(88.9 \%)$ & $9(11.1 \%)$ \\
DVIU + dilation & $58(31.5 \%)$ & $46(79.3 \%)$ & $12(20.6 \%)$ \\
\multicolumn{1}{c}{ Total } & $\mathbf{1 8 4}$ & $\mathbf{1 5 7}(\mathbf{8 5 . 3 \% )}$ & $\mathbf{2 7}(\mathbf{1 4 . 7 \% )}$ \\
\hline
\end{tabular}

Table 2. Number of previous DVIU and success rate.

\begin{tabular}{ccc}
\hline N. of previous DVIU & N. patients & Success rate \% \\
\hline 1 DVIU & 34 & $33(97.1 \%)$ \\
2 DVIU & 26 & $25(96.2 \%)$ \\
3 DVIU & 10 & $8(80 \%)$ \\
4 DVIU & 4 & $4(100 \%)$ \\
5 DVIU & 3 & $1(33.3 \%)$ \\
6 DVIU & 1 & $0(0 \%)$ \\
10 DVIU & 2 & $1(50 \%)$ \\
15 DVIU & 1 & $0(0 \%)$ \\
Total & $\mathbf{8 1}$ & $\mathbf{7 2 ( 8 8 . 9 \% )}$ \\
\hline
\end{tabular}


Table 3. number of previous DVIU + dilation and success rate.

\begin{tabular}{ccc}
\hline N. of previous DVIU + dilation & N. patients & Success rate \% \\
\hline 1 DVIU + dilation & 21 & $17(80.9 \%)$ \\
2 DVIU + dilation & 13 & $12(92.3 \%)$ \\
3 DVIU + dilation & 9 & $7(77.8 \%)$ \\
4 DVIU + dilation & 7 & $6(85.7 \%)$ \\
5 DVIU + dilation & 5 & $3(60 \%)$ \\
8 DVIU + dilation & 1 & $0(0 \%)$ \\
10 DVIU + dilation & 2 & $1(50 \%)$ \\
Total & $\mathbf{5 8}$ & $\mathbf{4 6}(\mathbf{7 9 . 3 \% )}$ \\
\hline
\end{tabular}

dilation and/or DVIU as a risk factor of failure in patients who undergoing open urethral reconstruction has not yet been fully investigated and reported in the literature. Culty and Boccon-Gibod reported that in patients who undergo anastomotic urethroplasty for post-traumatic urethral strictures, any previous urethral manipulation has a negative impact on the final outcome [15]. Waxman and Morey suggested that endoscopic treatment of urethral strictures using dilation or urethrotomy exacerbates scar formation, thus adding to stricture length and severity and complicating subsequent open repair [19]. Morey suggested that previous endoscopic manipulation has repeatedly been associated with higher failure rates following open urethroplasty [1]. Unfortunately, these authors do not provide any review in the literature or a personal study on a series of patients supporting this sentence, thus we can only suppose that this is a personal opinion without any supporting evidence $[1,19]$. In 2010, Breyer et al. reported a multivariate analysis of risk factors for long-term urethroplasty outcome in a large series of patients [17]. In this study, prior failed DVIU is reported as one of the factors predictive of failure after urethroplasty [17]. The authors investigated 443 patients who underwent urethroplasty, grouping together different stricture diseases by etiology (trauma or failed hypospadias repair), type of repair (anastomotic, graft, flap, combined) and length (from 1 to greater than $4 \mathrm{~cm}$ ) [17]. In so various a mixture of patients, it is really difficult to identify previous DVIU as the true cause of stricture recurrence. Urologists commonly increase their sample size by combining patients with different strictures, different etiologies, different locations and different surgical repair [20]. When performed in penile, bulbar or posterior tracts, or in strictures due to trauma, lichen sclerosus or failed hypospadias repair, the urethroplasty requires completely different surgical steps and presents completely different complication rates, and outcomes [20]. Although this approach may allow the data to reach a significant power, it fails to provide homogeneous groups [20].

One of the main strengths of our work is reporting on a group of homogeneous urethral conditions. We selected a homogeneous series of patients with the same stricture location (bulbar), (excluding those with panurethral strictures, traumatic strictures, lichen sclerosus, failed hypospadias repair, failed urethroplasty) treated by the same surgeon (GB) with the same standard surgical technique (ventral oral mucosal graft). The subgroups we identified presented similar characteristics as well, even if the number of patients enrolled in the single groups were different, since patients who had undergone more than 4 DVIU were not as numerous as patients who had undergone less than 4 DVIU (Tables 2 and $\mathbf{3}$ ).

The results of our present survey are similar to the results we previously reported in 2001, 93 patients underwent bulbar urethroplasty (27 end-to-end; 40 dorsal skin graft urethroplasty; 26 two-stage repair), and in 46 patients $(49 \%)$, the urethroplasty was performed as primary repair, and in $47(51 \%)$ the urethroplasty was performed after failed DVIU [16]. Primary urethroplasty showed an $85 \%$ success rate and urethroplasty after failed DVIU showed an $87 \%$ success rate [16]. The present survey in a more homogenous series of patients seems to confirm that failed DVIU does not influence the results of urethroplasty. Ventral onlay oral graft bulbar urethroplasty in 38 patients with no previous urethral instrumentation had an $86.8 \%$ success rate, and in 81 patients with previous DVIU had an $88.9 \%$ success rate (Table 1). The success rate of urethroplasty decreased to $79.3 \%$ in 58 patients with previous DVIU associated with dilation (Table 1). However, owing to the small size of some subgroups, we were not able to set a predictive model for urethroplasty outcome or to set a strong conclusions. Our study is not devoid of limitations. First of all, we recognized that our data may be considered evidence of a low level, due to the fact that external validity still needs to be checked out by our centre in international multicenter studies. In our present survey, the success rate of ventral onlay oral graft bulbar urethroplasty greatly decreases only when at least 4 endoscopic procedures had been previously performed (Tables $\mathbf{2}$ and $\mathbf{3}$ ), which seems to be in contrast with the current opinions reported in the literature $[1,7,15,19]$. But these preliminary data strongly require to be confirmed in a more large series of patients, because in our study the number of patients who had undergone more than 4 DVIU and dilation was so small (Tables 2 and 3) that it is difficult to compare with the large number of patients who had undergone less than 4 DVIU and dilation (Table 1). In the future, it would be interesting to investigate, in a largest series of patients, if other types of surgical techniques (end-to-end, augmented anastomotic repair, dorsal onlay graft urethroplasty) currently used in bulbar urethral reconstruction provide the same results in patients with a history of previously failed endoscopic treatment, as we reported in 2001 [16]. The main flaw of our study is a lack of any 
robust statistical analysis: however, we think that these preliminary data deserves attention. Periodic dilation and DVIU, despite the poor overall efficacy, still are the most common procedures used by the majority of urologists in the United States and urethroplasty rates were the lowest of all treatments [5-8]. The current literature suggests that urethral stricture should be considered an open surgical disease, because dilation and DVIU are now regarded as neither cost-effective nor efficacious as a long-term strategy [1]. The current trend is to reserve urethroplasty for patients in whom dilation or DVIU has failed. The question is if failed dilation or DVIU should be considered a risk factor for the outcome of urethroplasty.

In summary, in our preliminary survey, the role of dilation and DVIU as a risk factor of failure in patients who undergoing one-stage bulbar oral graft urethroplasty seems to be limited in patients presenting a history of more than 4 DVIU. But more studies including a largest series of patients are necessary to investigate the role of failed endoscopic procedures in the outcome of open surgery.

\section{REFERENCES}

[1] A. Morey, "Urethral Stricture Is Now an Open Surgical Disease," The Journal of Urology, Vol. 181, No. 3, 2009, pp. 953-954. doi:10.1016/j.juro.2008.12.026

[2] R. A. Santucci, "Should We Centralize Referrals for Repair of Urethral Stricture?" The Journal of Urology, Vol. 182, No. 4, 2009, pp. 1259-1260. doi:10.1016/j.juro.2009.06.108

[3] A. R. Mundy and D. E. Andrich, "Urethral Strictures," British Journal of Urology International, Vol. 107, No. 1, pp. 6-26. doi:10.1111/j.1464-410X.2010.09800.x

[4] R. Santucci and L. Eisemberg, "Urethrotomy Has a Much Lower Success Rate Than Previously Reported," The Journal of Urology, Vol. 183, No. 5, 2010, pp. 1859-1862. doi:10.1016/j.juro.2010.01.020

[5] T. L. Bullock and S. B. Brandes, "Adult Anterior Urethral Strictures: A National Practice Patterns Survey of Board Certified Urologists in the United States," The Journal of Urology, Vol. 177, No. 2, 2007, pp. 685-690. doi:10.1016/j.juro.2006.09.052

[6] J. T. Anger, V. C. Scott, C. Sevilla, M. Wang and E. M. Yano, "Patterns of Management of Urethral Stricture Disease in the Veterans Affairs System," Urology, Vol. 78, No. 2, 2011, pp. 454-458. doi:10.1016/j.urology.2010.12.081

[7] G. C. Ferguson, T. L. Bullock, R. E. Anderson, R. E. Blalock and S. B. Brandes, "Minimally Invasive Methods for Bulbar Urethral Strictures: A Survey of Membres of the American Urological Association," Urology, Vol. 78, No. 3, 2011, pp. 701-707. doi:10.1016/j.urology.2011.02.051

[8] J. T. Anger, J. C. Buckley, R. A. Santucci, S. P. Elliott, C. $\mathrm{S}$. Saigal and the Urologic Diseases in America Project, "Trends in Stricture Management among Male Medicare
Beneficiares: Underuse of Urethroplasty?" Urology, Vol. 77, No. 2, 2011; pp. 481-486. doi:10.1016/j.urology.2010.05.055

[9] V. Pansadoro and P. Emiliozzi, "Internal Urethrotomy in the Management of Anterior Urethral Strictures: LongTerm Follow-Up," The Journal of Urology, Vol. 156, No. 1, 1996, pp. 73-75.

[10] J. W. Steenkamp, C. F. Heynes and M. L. S. De Kock, "Internal Urethrotomy versus Dilation as Treatment for Male Urethral Strictures: A Prospective, Randomized Comparison," The Journal of Urology, Vol. 157, No. 1, 1997, pp. 98-101.

[11] C. F. Heynes, J. W. Steenkamp, M. L. S. De Kock and P. Whitaker, "Treatment of Male Urethral Strictures: Is Repeated Dilation or Internal Urethrotomy Useful?" The Journal of Urology, Vol. 160, No. 2, 1998, pp. 356-358.

[12] T. J. Greenwell, D. E. Castle, D. E. Andrich, J. T. MacDonald, D. L. Nicol and A. R. Mundy, "Repeat Urethrotomy and Dilation for the Treatment of Urethral Stricture Are Neither Clinically Effective nor Cost-Effective," The Journal of Urology, Vol. 172, No. 1, 2004, pp. 275-277. doi:10.1097/ju.0000132156.76403.8f

[13] J. L. Wright, H. Wessells, A. B. Nathens and W. Hollingworth, "What Is the Most Cost-Effective Treatment for 1 to $2 \mathrm{~cm}$ Bulbar Urethral Strictures: Societal Approach Using Decision Analysis," Urology, Vol. 67, No. 5, 2006 ; pp. 889-893. doi:10.1016/j.urology.2005.11.003

[14] K. F. Rourke and G. H. Jordan, "Primary Urethral Reconstruction: The Cost Minimized Approach to the Bulbous Urethral Stricture," The Journal of Urology, Vol. 173, No. 4, 2005, pp. 1206-1210. doi:10.1097/ju.0000154971.05268.81

[15] T. Culty and L. Boccon-Gibod, "Anastomotic Urethroplasty for Posttraumatic Urethral Stricture: Previous Urethral Manipulation Has a Negative Impact on the Final Outcome," The Journal of Urology, Vol. 177, No. 4, 2007, pp. 1374-1377.

[16] G. Barbagli, E. Palminteri and M. Lazzeri, "Long-Term Outcome of Urethroplasty after Failed Urethrotomy versus Primary Repair," The Journal of Urology, Vol. 165, No. 6, 2001, pp. 1918-1919.

[17] B. N. Breyer, J. W. McAninch, J. M. Whitson, M. L. Eisenberg, J. F. Mehdizadeh, J. B. Myers and B. B. Voelzke, "Multivariate Analysis of Risk Factors for LongTerm Urethroplasty Outcome," The Journal of Urology, Vol. 183, No. 2, 2010, pp. 613-617. doi:10.1016/j.juro.2009.10.018

[18] G. Barbagli, S. Sansalone, G. Romano and M. Lazzeri, "Ventral Onlay Oral Mucosal Graft Bulbar Urethroplasty," British Journal of Urology International, Vol. 108, No. 7, pp. 1218-1231. doi:10.1111/j.1464-410X.2011.10625.X

[19] S. W. Waxman and A. F. Morey, "Management of Urethral Strictures," The Lancet, Vol. 367, No. 9520, 2006, pp. 1379-1380.

[20] G. Barbagli and M. Lazzeri, "Can Reconstructive Urethral Surgery Proceed Without Randomized Controlled Trials?" European Urology, Vol. 54, No. 4, 2008, pp. 709-711. doi:10.1016/j.eururo.2008.03.010 